\title{
Entanglement of a Gas of Atomic Spins
}

\author{
N.P. BigELOW \\ Department of Physics and Astronomy, Institute of Optics \\ and Laboratory for Laser Energetics \\ The University of Rochester, Rochester, NY 14627, U.S.A. \\ In this paper we discuss our recent work on the creation of massively \\ entangled states of millions to trillions of thermal atomic spins. We consider \\ the particular entangled states that are also spin-squeezed states. \\ PACS numbers: 03.75.-b, 42.50.- $\mathrm{p}, 32.80 .-\mathrm{t}$
}

\section{Introduction}

Entanglement is one of the most intrinsically quantum aspects of the quantum theory. Entanglement is of importance to many advanced quantum technologies such as quantum computers and teleportation devices. As a result, the entanglement of many atoms has developed into an important frontier of contemporary physics and quantum optics. In this paper we describe our work on the creation of entanglement via the creation of spin-squeezed states of a gas of neutral atoms. In our work we have chosen to emphasize spin-noise and spin-squeezing [1, 2] as our focal point and our "detector" of entanglement for several reasons. First, the collective spin-state of an atomic gas is comparatively easy to probe. Second, because spin-noise has important consequences for precision measurements and third, because of the relevance of collective continuous variables such as spin, to massive particle teleportation.

To appreciate the practical importance of spin-squeezing, consider the case of a neutral atom atomic clock: a Ramsey interferometer. The many-atom entangled, spin-squeezed state (SSS) can be used to dramatically improve the measurement sensitivity of a typical interferometer from $1 / \sqrt{N}$ to $1 / N$ where $N$ is the number of detected atoms. At first glance one might guess that the limit of quantum noise in atomic clocks is far from important, making spin-squeezing mostly of academic interest. However, this fundamental barrier for the cold-atom "fountain" clocks has been observed and is known as the clock's "projection-noise" [3]. 
Historically, there has been an extensive research activity devoted to the generation of the non-classical states of the electro-magnetic field and to the nature of entanglement of pairs of photons generated, for example, in parametric down conversion [4]. Indeed, for the case of photon states of the electromagnetic field, these ideas have resulted in the successful experimental demonstration of quantum state teleportation [5]. With a surge of interest in new quantum technologies based on such controlled entanglement, this effort is now being paralleled by a quest for similar entanglement of massive particle systems. Indeed, protocols for spin-state teleportation have already been described [6]. In one particularly highly publicized paper [7] a group has used a technique analogous to the method that we describe here [8-10] to entangle a gas of atoms partitioned in two closely spaced glass cells. For this paper we focus only on work carried out in our laboratories [8-10] and emphasize how we have used our apparatus to realize non-classical states of the collective atomic spin of the gas.

\section{The coherent spin-state and the spin-squeezed state}

For the spin polarized state of the sample, the collective spin of the system is along the alignment axis and is a minimum (spin) uncertainty state referred to as a coherent spin-state (CSS) (see Fig. 1) [11].

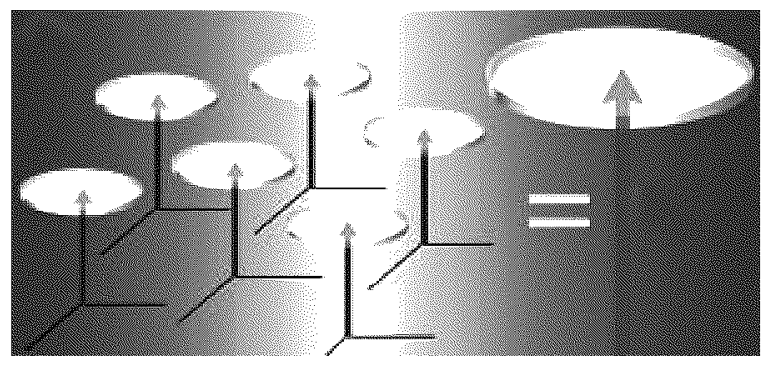

Fig. 1. For the case of six individual spins that are all aligned along the same axis, the sample is in a coherent-spin state which is a minimum uncertainty state.

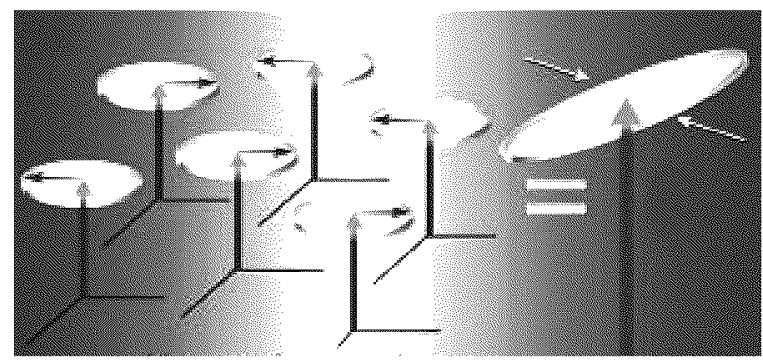

Fig. 2. When the spins are entangled, the collective spin is squeezed. 
The CSS is a state in which atoms of spin $F$ are completely in the state with magnetic projection $m_{F}=F$ (i.e. $|\Psi\rangle=\left|F=F, m_{F}=F\right\rangle$ ). When the spins of the system are fully correlated - when they are entangled - then the collective spin uncertainty along one particular axis is reduced below the (uniform) transverse uncertainty of the CSS (see Fig. 2).

It is now widely recognized that the use of squeezed atomic states has the potential for substantial improvement in the sensitivity of atom interferometers such as the two-zone Ramsey atomic clock and the atomic fountain clock. This realization has led to diverse theoretical and experimental work on schemes to realize atomic spin-squeezing. Building upon the seminal work on photon number squeezing by Kitagawa and Yamamoto [12] Kitagawa and Ueda proposed and clarified the basic issues concerning the definition and preparation of spin-squeezed states. This was followed by studies of the production of squeezed atomic states $[1,2]$, the transfer of squeezing from incident squeezed light to an assembly of excited state cold atoms, and the measurements related to a collection of squeezed atoms $[8-10,13-16]$

\section{Measuring the spin-state and spin-noise using paramagnetic Faraday rotation}

In order to use spin-squeezing as a measure of entanglement, we must understand the measurement of atomic spin (projection) noise. In a variety of experiments in our group and elsewhere, quantum spin-noise has already been investigated $[8,13]$.

Our underlying technique is based on the direct measurement of the rotation of the polarization axis of a nearly-resonant linearly polarized laser field passing though an atomic vapor $[8,9,14,15]$ as shown in Fig. 3 .

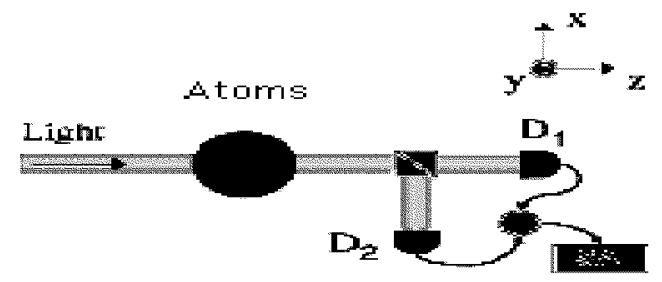

Fig. 3. Experimental set-up for measurement of the paramagnetic Faraday rotation and of the collective atomic spin. A linearly polarized laser field interacts with an atomic sample and the rotation of the plane of polarization is detected using a polarization sensitive beam splitter and two detectors $\left(D_{1}\right.$ and $\left.D_{2}\right)$.

In this set-up, a collimated laser beam, linearly polarized at a $45^{\circ}$ angle between the $\boldsymbol{x}$ and $\boldsymbol{y}$ axes is passed through an atomic sample. For atoms with ground state angular momentum of $\hbar / 2$, in an off resonant atom-photon interaction, the 
interaction Hamiltonian scales as $\boldsymbol{s}_{z}(t) F_{z}(t)$, where $F$ is the $z$-projection of the collective atomic spin $\left(\boldsymbol{F}_{z}(t)=\sum_{j} \boldsymbol{F}_{z}^{j}\right.$, where $\boldsymbol{F}_{z}^{j}$ is an individual atomic spin $)$ and where $\boldsymbol{s}_{z}$ is the photon spin operator defined as $\boldsymbol{s}_{z}=(1 / 2)\left[a_{\mathrm{v}}^{\dagger}(t) a_{\mathrm{h}}(t)+a_{\mathrm{h}}^{\dagger}(t) a_{\mathrm{v}}(t)\right]$ (here the $a$ 's are creation and annihilation operators for horizontally and vertically polarized probe field modes). In detail, we describe the optical field in terms of the Stokes operators of the field and as the atom number becomes large, we replace the sums with integrals and redefine our operators and variables as continuous quantum variables.

The physical effect of interest is that the polarization state of the forward scattered light is rotated by an amount which depends on the value of the collective atomic spin $\boldsymbol{F}_{z}(t)$. From the experimental view point, what is key is that the noise on the rotation angle is influenced by the atomic (quantum) spin-noise. In this polarimeter, after the light passes through the sample, it is projected onto particular vertical and horizontal axes by a polarization sensitive beam-splitter cube (a Glan-Thompson beam splitter) and detected by photo-detectors $D_{1}$ and $D_{2}$. It is the signal generated by the two detectors that is used to determine the orientation of the collective atomic spin, and, for our work, to determine the atomic spin-noise.

We stress that as we are interested in spin-fluctuations we focus primarily on the "spin-noise" in the Faraday rotation measurement. That is, we focus on the fluctuations in the rotation angle. Using our polarimeter, we measure these fluctuations in either of two modes. In the first mode, pulse trains composed of individual pulses of duration $2-300 \mathrm{~ns}$ are passed through the atomic sample. As the pulses arrive at detectors $D_{1}$ and $D_{2}$ the resulting signals are digitized and recorded by a computer. Every 10,000 or so pulses, the difference signal $\left(D_{1}-D_{2}\right)$ is

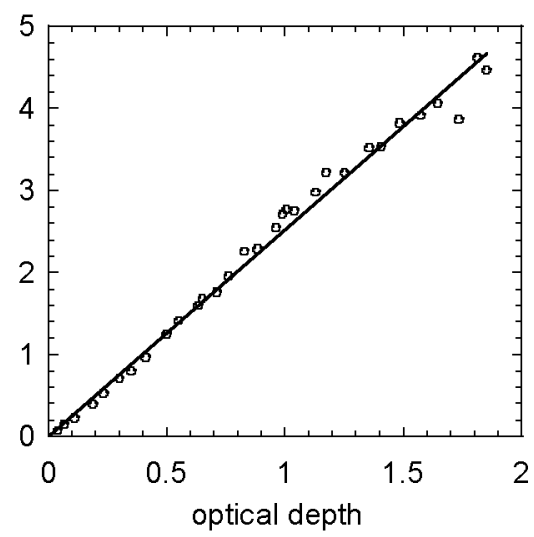

Fig. 4. Measured spin-noise as a function of optical thickness of the sample. The linear dependence of the noise on optical depth, and hence atom number, is a signature of atomic shot-noise $[8,13,15]$. The spin-noise is arbitrary units and the optical thickness is a dimensionless variable. 
calculated and the variances calculated. In a second operation mode, a continuous $(\mathrm{CW})$ laser beam is passed through the sample. To examine the noise, the difference current from the detectors, again $\left(D_{1}-D_{2}\right)$, is found using a balanced difference amplifier, and the noise spectrum is measured using a high-performance spectrum analyzer.

In our first set of experiments [8], we demonstrated that we could isolate the noise contribution in the polarimeter due to the atomic shot-noise. In this work, the measurements were made using an unpolarized (thermal) atomic sample. Measured as a function of the optical thickness of the sample (see Fig. 4), we observed a spin-noise that scaled linearly with the number of atoms in the interaction zone (defined by the sample temperature and the profile of the probe laser beam). This provided a clear signature that we had reached the quantum limit in the spin noise measurement; the atomic "spin-shot-noise".

\section{Obtaining and detecting entanglement: spin-squeezing}

The realization of ground-state spin-squeezing in a vapor of cesium atoms was first demonstrated in our laboratories [10]. This spin-squeezing can be understood as a particular case of quantum entanglement in which the correlations between spins are introduced in such a way that the effect is to reduce fluctuations associated with projection noise in the measurement of the collective atomic spin of the vapor (see Fig. 2). We prepared the spin-squeezed sample by first state-selecting the atomic vapor to prepare a completely polarized sample in a coherent spin-state which represents a minimum (spin) uncertainty spin-state of the sample [11]. The state was then "spin-squeezed" using a so-called QND-type (quantum non-demolishing) interaction provided by the laser-atom coupling [9]. The action of a QND measurement as a state-preparation device is well known. However, in our case, the creation of entanglement can also be viewed as resulting from a non-local Bell-type measurement of the EPR operators, formed from the Stokes operators of the light field, after interaction with the atomic system [6]. Schematically, this process is characterized in Fig. 5.

Under these conditions the collective spin-state of the system was caused to undergo a slow oscillation around the polarization axis (the axis of the collective spin) and the noise in the measurement of the rotation angle of the light was evaluated. We note that our choice to examine the noise in the rotation angle measurement is not at all restrictive. This measurement is the direct analogue of a large class of interferometer-type experiments such as performed in a cold-atom fountain or in an EDM experiment (an experiment that searches for the permanent dipole moment of the electron).

To create the CSS, we optically pumped the atomic sample using two additional diode lasers. The atomic sample was a cesium vapor (saturated vapor pressure at $300 \mathrm{~K}$ ) contained in a glass cell whose inner walls were coated with high molecular-weight paraffin. This coating minimized the atomic spin dephas- 


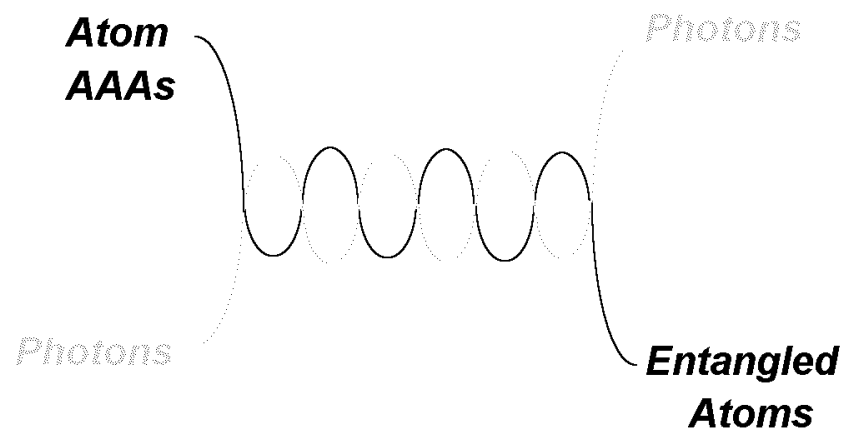

Fig. 5. Measurement of the photon state of the light beam exiting the polarimeter serves as a state preparation device for the atomic system. What is notable is that by making such a non-local Bell type measurement, a non-classical state of the atomic ensemble can be created using coherent (classical) light. This is distinctly different from previous spin-squeezing work [15] on excited state cesium where non-classical light was vital to achieving squeezing.

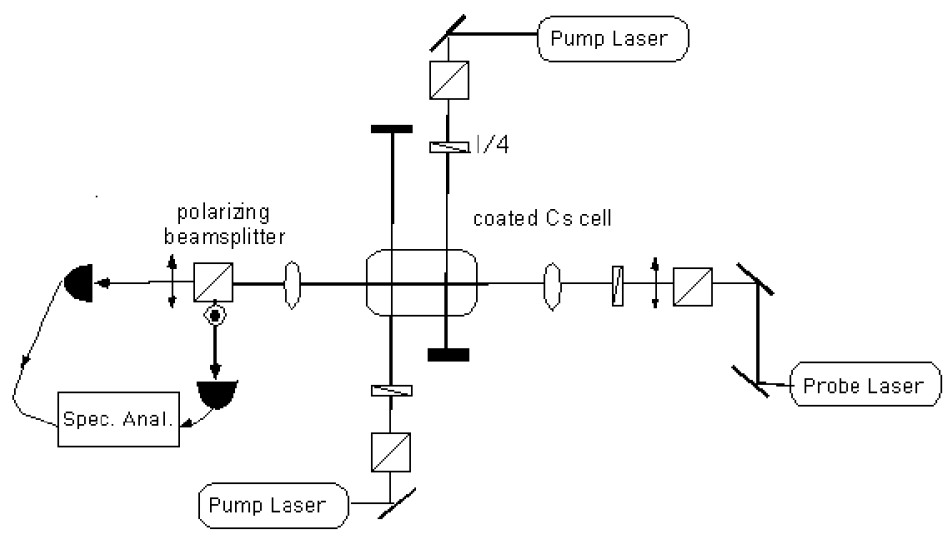

Fig. 6. Modified polarimeter apparatus. Two additional lasers are used to pump the sample into a CSS. In this measurement the spin-noise was examined using a CW probe laser and a low-frequency spectrum analyzer.

ing at the walls, permitting long spin lifetimes ( $\sim$ minute) and high ground state polarization $(\sim 95 \%)$. The modified polarimeter apparatus is shown in Fig. 6.

In Fig. 7 we show the noise spectrum generated from the difference current of the two photo-detectors shown in the polarimeter set-up above (Fig. 5). The dashed line is the atomic shot-noise level that defines the "standard quantum limit" (SQL) for the measurement. The sharp peak at $16 \mathrm{MHz}$ is due to the $\mathrm{RF}$ field used to cause the spin oscillations. Note that the noise floor is well below the ("unsqueezed") projection-noise level of the sample. These data clearly show a $75 \%$ spin-noise reduction below the SQL, which, for a polarization of $95 \%$, corresponds to $70 \%$ spin-squeezing [10]. 


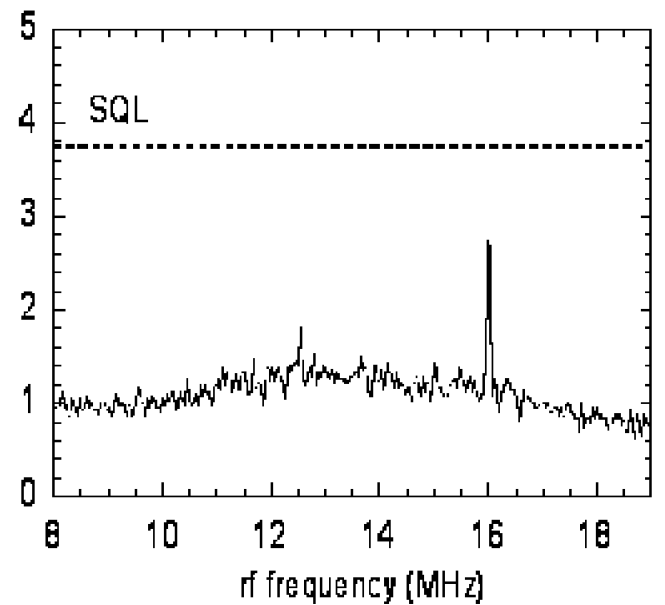

Fig. 7. Noise spectrum of the SSS sample. The dashed line shows the SQL defined by the atomic shot-noise level of the unsqueezed sample. The vertical scale is arbitrary noise (variance) units. The sharp peak is the RF signal used to cause the spin oscillation about the polarization axis measured in our spin-interferometer (see the text) [10].

In the case where the measurements were made as a series of paired pulses [8] one can choose to view the first pulse as a state-preparation pulse and the second pulse as the read-out pulse. However, the fact that experiments can also be performed in a continuous (CW) fashion [10], points to the underlying fact that the overall evolution of the quantum state in the interaction + measurement process is what is fundamental to creating entanglement.

When measurements are made using the apparatus in a pulsed mode, we can gain insight into the microscopics of the experiment. In particular, when we perform the spin-noise measurement using a pair of pulses, we find that quantum correlations in the measurement decay if the pulses are separated by times comparable to the atomic transit time through the laser beam. We take this as a proof that the correlations and entanglement are not created by atom-atom interactions [17], but are instead created by the interaction of the collective spin with the optical field. In concept, the atoms could be spaced by a very large distance, and entanglement could still be realized. This point was at the heart of the work of Ref. [13].

Recently, unconditional quantum state teleportation between separated atomic ensembles has been discussed in the context of continuous quantum variables $[6,18]$. Although our sample does not fit the most familiar description of a separated ensembles, if we think of the sample as composed of a series of slabs of atoms frozen in space, the language of state teleportation can be used to shed some light on the more complex case of our $\mathrm{CW}$ experiment. Consider with the first and second slabs. We are performing a non-local Bell-type measurement which 
entangles the two ensembles. Then, as the "measurement continues", we make a Bell measurement which teleports the state of ensemble 2 onto ensemble 3 , and so one, entangling the entire sample. In this description, the teleportation fidelity is very high because there is little transmission loss as the light field propagates from slab to slab, with the dominant source being spontaneous emission noise [6]. From an experimental point of view, this teleportation description becomes more natural when we recognize that a continuous signal, when detected and analyzed in the spectrum analyzer, is effectively broken into time intervals which are then correlated in generating the noise spectrum (i.e. there is an underlying time scale set by the spectrum analyzer's resolution bandwidth).

The work described in this paper reflects a collaboration and this paper is dedicated to one of the collaborators: Professor Leonard Mandel. Particular thanks is due to Dr. Alex Kuzmich. This work was supported by the National Science Foundation, the Office of Naval Research and the Army Research Office.

\section{References}

[1] M.A. Rashid, J. Math. Phys. 19, 1391 (1978); 19, 1397 (1978); D.F. Walls, P. Zoller, Phys. Rev. Lett. 47, 709 (1981); K. Wodkiewicz, J. Eberly, J. Opt. Soc. Am. B 2, 458 (1985); M. Kitagawa, M. Ueda, Phys. Rev. Lett. 67, 1852 (1991); M. Kitagawa, M. Ueda, Phys. Rev. A 47, 5138 (1993).

[2] D.J. Wineland, J.L. Bollinger, W.M. Itano, F.L. Moore, D.J. Heinzen, Phys. Rev. A 46, R6797 (1992); D.J. Wineland, J.J. Bollinger, W.M. Itano, D.J. Heinzen, Phys. Rev. A 50, 67 (1994).

[3] G. Santarelli, Ph. Laurent, P. Lemonde, A. Clairon, A.G. Mann, S. Chang, A.N. Lutin, C. Salomon, Phys. Rev. Lett. 82, 4619 (1999).

[4] see for example L. Mandel, E. Wolf, Optical Coherence and Quantum Optics, Cambridge University Press, Cambridge (England) 1995.

[5] D. Bouwmeester, J.-W. Pan, K. Mattle, M. Eibl, H. Weinfurter, A. Zeilinge, Nature 390, 575 (1997); D. Boschi, S. Branca, F. De Martini, L. Hardy, S. Popescu, Phys. Rev. Lett. 80, 1121 (1998); A. Furusawa, J.L. Sørensen, S.L. Braunstein, C.A. Fuchs, H.J. Kimble, E.S. Polzik, Science 282, 706 (1998).

[6] L.M. Duan, J.I. Cirac, P. Zoller, E.S. Polzik, Phys. Rev. Lett. 85, 5643 (2000).

[7] B. Julsgaard, A. Kozhekin, E. Polzik, Nature 413, 400 (2001).

[8] A. Kuzmich, L. Mandel, J. Janis, Y.E. Young, R. Ejnisman, N.P. Bigelow, Phys. Rev. A 60, 2346 (1999).

[9] A. Kuzmich, N.P. Bigelow, L. Mandel, Europhys. Lett. 42, 481 (1998).

[10] A. Kuzmich, L. Mandel, N.P. Bigelow, Phys. Rev. Lett. 85, 1594 (2000).

[11] J. Klauder, Ann. Phys. (N.Y.) 11, 123 (1960); J.M. Radcliffe, J. Phys. A 4, 313 (1971); F.T. Arecchi, E. Courtens, R. Gilmore, H. Thomas, Phys. Rev. A 6, 2211 (1972).

[12] M. Kitagawa, Y. Yamamoto, Phys. Rev. A 34, 3974 (1986). 
[13] E.B. Alexandrov, V.S. Zapasski, Sov. Phys. JETP 54, 64 (1981); A.M. Bacon, H.Z. Zhao, L.J. Wang, J.E. Thomas, Phys. Rev. Lett. 75, 1296 (1995); W.M. Itano, J.C. Bergquist, J.J. Bollinger, J.M. Gilligan, D.J. Heinzen, F.L. Moore, M.G. Raizen, D.J. Wineland, Phys. Rev. A 47, 3554 (1993); J.L. Sørensen, J. Hald, E.S. Polzik, J. Mod. Opt. 44, 1917 (1997).

[14] Y. Takahashi, K. Honda, N. Tanaka, K. Toyoda, K. Ishikawa, T. Yabuzaki, Phys. Rev. A 60, 4974 (1999).

[15] A. Kuzmich, K. Mølmer, E.S. Polzik, Phys. Rev. Lett. 79, 4782 (1997); J. Hald, J.L. Sørensen, C. Schori, E.S. Polzik, Phys. Rev. Lett. 83, 1319 (1999).

[16] H. Saito, M. Ueda, Phys. Rev. Lett. 79, 3869 (1997).

[17] S. Osnaghi, P. Bertet, A. Auffeves, P. Maioli, M. Brune, J.M. Raimond, S. Haroche, Phys. Rev. Lett. 87, 037902 (2001); D. Jaksch, C. Bruder, J.I. Cirac, C.W. Gardiner, P. Zoller, Phys. Rev. Lett. 81, 3108 (1998); D. Jaksch, H.-J. Briegel, J.I. Cirac, C.W. Gardiner, P. Zoller, Phys. Rev. Lett. 82, 1975 (1999).

[18] L.M. Duan, G. Giedke, J.I. Cirac, P. Zoller, Phys. Rev. Lett. 84, 2722 (2000); S. Braunstein, H.J. Kimble, Phys. Rev. A 61, 042302 (2000). 